\title{
Original
}

\section{The Growth Kinetics of Salmonella Enteritidis in Raw Ground Beef}

\author{
ISLAM I. SABIKE ${ }^{1,2}$, HIROSHI FUJIKAWA $^{1 *}$, AND ABOBAKR M. EDRIS $^{2}$ \\ ${ }^{1}$ Laboratory of Veterinary public health, Faculty of Agriculture, Tokyo University of \\ Agriculture and technology, Japan. \\ ${ }^{2}$ Department of Food control, Faculty of veterinary medicine, Benha University, Egypt
}

Received 9 December, 2014/Accepted 18 February, 2015

\begin{abstract}
The growth kinetics of Salmonella Enteritidis in raw beef has been little studied so far. Thus, this study aimed to clarify the growth kinetics of the pathogen in ground beef using a growth model. When Salmonella cells inoculated at various initial doses into ground beef were incubated at a given temperature $\left(24^{\circ} \mathrm{C}\right)$, the maximum population $\left(N_{\max }\right)$ of the microbe at the stationary phase varied with the doses. This relationship was expressed with a polynomial equation for $\boldsymbol{N}_{\max }$ using the initial dose. The combination of the growth model and the polynomial equation successfully predicted Salmonella growth at a given initial dose. When Salmonella cells inoculated in ground beef were incubated at various constant temperatures, the growth curves of the pathogen and natural microflora (NM) were well described with the growth model. The rate constant of growth and the $\boldsymbol{N}_{\max }$ values for Salmonella and NM were then analyzed kinetically. From these results, growth curves of Salmonella and NM in ground beef stored at dynamic temperatures were successfully predicted. Competition between Salmonella and NM in ground beef was also found during the storage. This study could give usable information on the growth of Salmonella and NM in ground beef at various temperatures.
\end{abstract}

Key words : Salmonella / Ground beef / Logistic model / Polynomial model / Growth kinetics.

\section{INTRODUCTION}

Salmonella has been estimated to cause 93.8 million human infections (food-borne cases were estimated to be 80.3 million) and 155,000 deaths annually worldwide (Majowicz et al., 2010). Especially, Salmonella Enteritidis has been ranked at the top of the fifteen most common Salmonella serovars isolated from humans in 37 countries between 2001 and 2007 and between 2010 - 2014 in Japan (Hendriksen et al., 2011; IDSC, 2014).

Beef is one of the most commonly implicated food commodities in the outbreaks of Salmonella infections (accounting for $8 \%$ of the outbreaks). Salmonella Enteritidis is one of the most common serotypes causing beef-associated outbreaks (accounting for 18\%) (Jackson et al., 2013). It has been estimated

*Corresponding author. Tel \& Fax: +81-42-367-5598, E-mail: fujik (a)cc.tuat.ac.jp that the prevalence of Salmonella in ground beef was $1.9 \%$, but $1.1 \%$ in intact raw meat (USDA, 2012). This high prevalence in ground beef imposes a high risk of salmonellosis compared with larger pieces of beef. Actually, ground beef can be contaminated throughout its preparation process due to the increased surface area used and mixing done during the grinding operation (Ayres, 1955). Also, inadequate cleaning and sanitization of meat grinder resulted in sustained Salmonella contamination of ground beef (Roels et al., 1997). Three multistate outbreaks of Salmonella infections were linked to ground beef in the United States in 1994, 2004 and 2013 (CDC, 1995, 2006, and 2013). Although ground beef is less commonly a vehicle for Salmonella Enteritidis than other meats like poultry (Arthur et al., 2008), one multistate outbreak occurred due to the consumption of ground beef contaminated with Salmonella Enteritidis in the United States in 2012 (CDC, 2012). The presence of any Salmonella serovar is potentially a health threat. 
Therefore an effective strategy to control food-borne salmonellosis needs to be made with the knowledge of the growth kinetics of the pathogen in ground beef. This approach is named predictive microbiology (McMeekin et al., 1993). Predictive mathematical models can be used to evaluate the change in pathogen density at any given time and according to the temperatures during storage and distribution. As a result, the models enable users to evaluate the effect of processing, distribution, and storage operations on the microbiological safety and quality of foods (McMeekin et al., 1993). As such, it can provide insight on the shelf life or safety of foods (D’Aoust, 1991).

Among many environmental factors affecting the Salmonella growth in ground beef, temperature is one of the major factors that can influence microbial growth during its passage through the food chain. Mackey and Kerridge (1988) studied mathematically the relationship between the growth rate of Salmonella in minced beef and temperature using the square root model (Ratkowsky et al., 1982). However, it was a simple study with only two levels of Salmonella inoculum and the temperature patterns examined were only isothermal temperatures (Mackey and Kerridge, 1988). Juneja et al. (2009) studied the growth rate of Salmonella in ground beef, but at constant temperatures in irradiated ground beef. Therefore, their study lacked factors found in the real environment for food, namely the interaction between natural microflora (NM) and Salmonella. Moreover, all growth curves obtained in their study did not contain the stationary phases, and the rate constant of growth and the lag period were only kinetically studied (Juneja et al., 2009). Thus, it is not likely that the growth kinetics of the pathogen in raw ground beef has been fully studied so far.

Since Salmonella Enteritidis often contaminates chicken and eggs and has caused serious food poisoning outbreaks worldwide, the authors (Zaher and Fujikawa, 2011; Sakha and Fujikawa, 2012 and 2013) studied the growth of Salmonella Enteritidis in raw ground chicken and the commercial products of pasteurized and unpasteurized liquid egg with a growth model extended from the logistic model (Fujikawa et al., 2003 and 2004). The growth model successfully predicted the Salmonella growth in raw ground chicken and the liquid egg products at dynamic temperatures (Zaher and Fujikawa, 2011; Sakha and Fujikawa, 2012 and 2013).

In the present study, therefore, the growth of Salmonella Enteritidis in raw ground beef under conditions of various initial doses of the pathogen and temperature was kinetically studied with our model. The relationship between the pathogen and NM in ground beef during storage was also studied.

\section{MATERIALS AND METHODS}

\section{Salmonella cell preparation}

Four Salmonella Enteritidis strains SE2, SE3,SE5 and 04-137, which were all studied in our previous study with the liquid egg products (Zaher and Fujikawa, 2011; Sakha and Fujikawa, 2012 and 2013), were activated on XLD agar plates (Oxoid, Basingstoke, UK) at $37^{\circ} \mathrm{C}$ for $24 \mathrm{~h}$. Cells of several well-grown colonies on the plate were incubated in Trypticase soy broth (Oxoid) with shaking at $110 \mathrm{rpm}$ and at $37^{\circ} \mathrm{C}$ for $24 \mathrm{~h}$. Cultured cells ( $1 \mathrm{ml}$ each) were washed with saline $(0.85 \%(\mathrm{w} / \mathrm{v})$ sodium chloride solution) by centrifugation at $13,000 \times g$ and at $4^{\circ} \mathrm{C}$ for $15 \mathrm{~min}$. Cells were thoroughly suspended in saline $(1 \mathrm{~mL})$, yielding a cell suspension of about $10^{9} \mathrm{CFU} / \mathrm{ml}$. A cocktail of the four Salmonella suspensions with equal volumes was then made. In the experiment at different initial cell doses, the cell suspension was diluted to corresponding ratios with saline.

\section{Ground beef}

Ground beef $(10 \mathrm{~kg})$ was purchased at a retail store in Tokyo and examined for contamination with Salmonella sp. with the conventional method (Anonymous, 2004). After thorough mixing, samples of about $250 \mathrm{~g}$ were placed in sterile plastic bottles. The bottles were frozen at $-40^{\circ} \mathrm{C}$ until use and then thawed at $<10^{\circ} \mathrm{C}$ overnight for use.

\section{Salmonella inoculation and storage}

Ground beef samples were inoculated with the pathogen and stored in the same manner as had been used for ground chicken and liquid egg products (Zaher and Fujikawa, 2011; Sakha and Fujikawa, 2012 and 2013). Briefly, ground beef samples were inoculated with the Salmonella cell suspension prepared above (2 $\mathrm{ml} / 100 \mathrm{~g}$ beef). After thorough mixing with a sterile stainless steel spoon, 10-g portions were placed in sterile glass bottles (vacant volume $110 \mathrm{ml}$ ) with tight caps. The bottles were then stored at a constant temperature. Immediately after incubation, each sample (one bottle per data point) was taken from the incubator and cooled in ice water. Three trials were performed at a constant temperature. For the dynamic temperature experiment, the glass bottles were placed in a programmable incubator and the temperature at the center of each sample was measured in triplicate every $30 \mathrm{~s}$ throughout the experiment with a digital thermometer. Immediately after each incubation period, the sample in triplicate was taken from the incubator and cooled in ice water. 


\section{Bacterial cell counts}

Bacterial cell counts in the ground beef samples were done in the same manner as had been done for ground chicken and liquid egg products (Zaher and Fujikawa, 2011; Sakha and Fujikawa, 2012 and 2013). Briefly, the beef samples in the bottles were mixed with a buffered sodium chloride peptone solution to make $10 \%$ food homogenates in filtered plastic bags. After stomaching, the sample homogenate was serially 10-fold diluted with saline (Anonymous, 2004). Total (aerobic) bacteria counts of the sample were enumerated in duplicate with the surface-plating method using standard method agar plates (Nissui Pharmaceuticals) (Anonymous, 2004). Salmonella counts of the sample were enumerated in duplicate with the surface-plating method using XLD agar plates. Suspected colonies were examined for identification with a serological test using antiserum to Salmonella O antigens (DenkaSeiken, Tokyo) on a glass slide. The counts of NM were calculated by subtracting the Salmonella counts from the total bacteria counts for each sample. For the original ground beef without Salmonella inoculation, the NM counts were those of total bacteria counts with standard method agar plates. The average count with two plates was obtained for NM and Salmonella for each data point.

Salmonella cells at a very low dose were enumerated with the 5-tube most probable number (MPN) method (Anonymous, 2004). Namely, 3 dilutions consisting of 10,1 , and $0.1 \mathrm{~mL}$ of a $10 \%$ food homogenate of a sample were cultured in each 5 tubes containing Enterobacteriaceae Enrichment Mannitol broth (Merck, Darmstadt, Germany) and then isolated on XLD plates. Suspected colonies were then tested as described above.

\section{Growth model}

Averages of bacteria counts for the three trials of the constant temperature experiments or the three samples of the dynamic temperature experiments were calculated for analysis (Zaher and Fujikawa, 2011; Sakha and Fujikawa, 2012 and 2013). Salmonella and total bacteria counts of the samples during the storage were then analyzed with the extended logistic model, which is expressed as follows (Fujikawa et al., 2003 and 2004).

$$
\frac{d N}{d t}=r N\left\{1-\left(\frac{N}{N_{\max }}\right)^{m}\right\}\left\{1-\left(\frac{N_{\min }}{N}\right)^{n}\right\}
$$

Here $N$ is the population of a microorganism (CFU $/ g$ ) at time $t(h), r$ is the rate constant of growth $(1 / \mathrm{h}), N_{\max }$ is the maximum population $(\mathrm{CFU} / \mathrm{g})$, and $N_{\min }$ is the initial population (CFU/g). $m$ and $n(>0)$ are parameters related to the curvature of the deceleration phase and the period of the lag phase, respectively. The equation was solved numerically with the 4th-order Runge-Kutta method. Numerical data of microbial counts were analyzed by a computer program to fit to the growth model, which was developed using a spreadsheet software program, Microsoft Excel (Fujikawa and Kano, 2009). Here microbial populations estimated by the model (CFU/g) were then transformed to logarithms to make a growth curve.

The value for $N_{\max }$ of a Salmonella growth curve at a given temperature was estimated with a third-order polynomial equation with the initial population $(I)$ of Salmonella. The value for $N_{\max }$ under a given condition of the initial population (I) of Salmonella and temperature $(T)$ was also estimated with a polynomial equation of the third order. The parameters in these polynomial equations were obtained with the Solver function in Excel.

\section{Statistical analysis}

Performance of a mathematical model was evaluated with the square root of the mean of the square error, RMSE between log-transformed cell concentrations was estimated with the model $\left(\log N_{\text {est }}\right)$ and those observed $\left(\log N_{\text {obs }}\right.$ ) at the observation points, which is described below.

$$
R M S E=\sqrt{\frac{\sum_{i=1}^{k}\left(\log N_{\text {iobs }}-\log N_{\text {iest }}\right)^{2}}{k}}
$$

Here $k$ is the number of observation points. Statistical analyses of data including regression analyses and the t-test were performed with Microsoft Excel.

\section{RESULTS}

\section{Growth kinetics at various initial doses}

Growth kinetics of Salmonella Enteritidis (a cocktail of four strains) in raw ground beef at various initial doses of the pathogen was first studied. Ground beef was originally contaminated with $5.7 \mathrm{log} \mathrm{CFU} / \mathrm{g}$ of NM, but free of Salmonella. Salmonella was injected into ground beef at various initial doses ranging from 2.3 to $5.3 \log \mathrm{CFU} / \mathrm{g}$ and then stored at the given temperature of $24^{\circ} \mathrm{C}$. Salmonella growth curves in ground beef were similar during the storage (Fig. 1A). The growth curves in the figure were precisely described with the growth model. The values of the rate constant of growth $(r)$ were similar among them, ranging from 0.60 to 0.70 (1/ h). Also, the lag periods in the growth curves were also similar, ranging from 1.6 to $3.1 \mathrm{~h}$. However, the $N_{\max }$ value in the stationary phase was clearly higher with higher initial doses. Values for $N_{\max }$ were 7.2, 8.3, 8.8, 

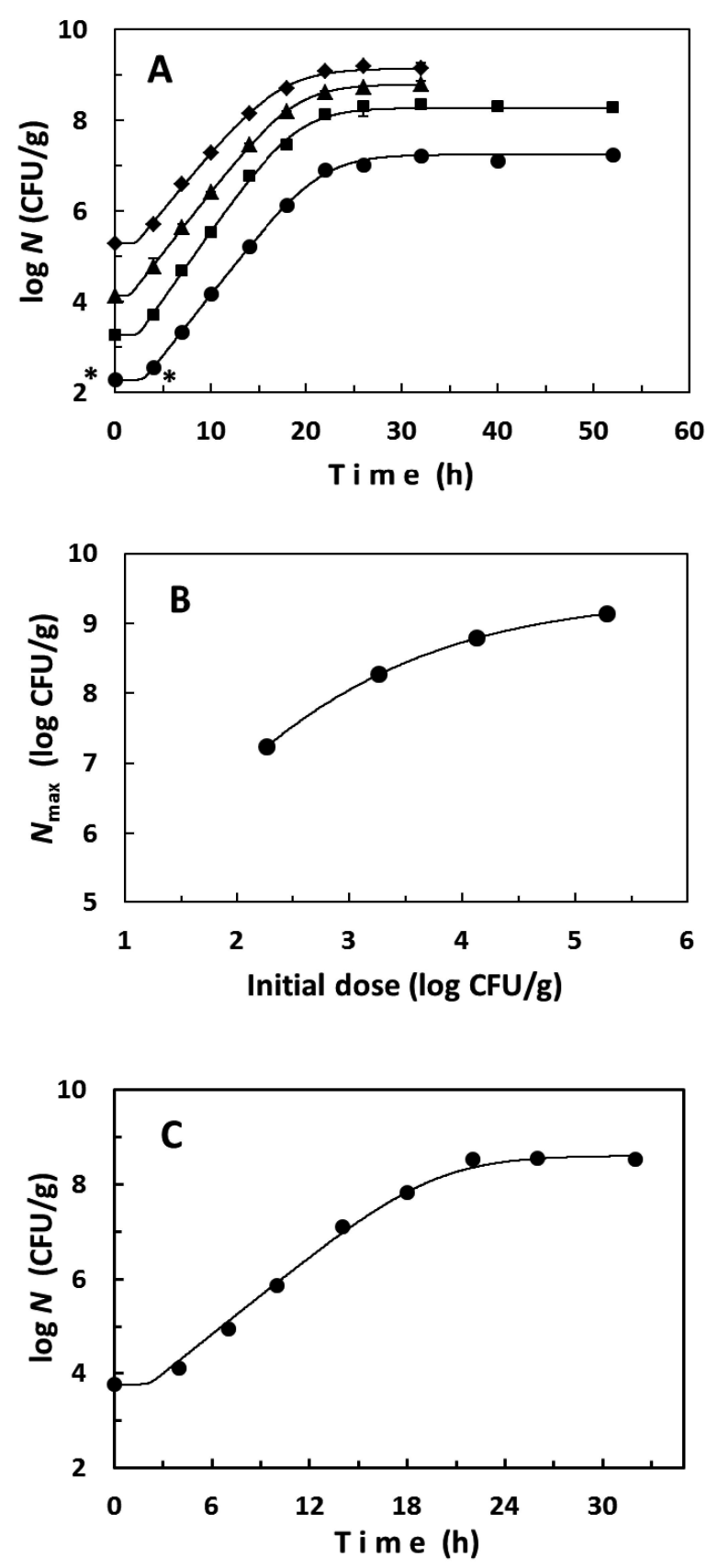

FIG. 1. Growth of Salmonella Enteritidis in raw ground beef at various initial doses.

A. Growth curves of the pathogen with initial doses ranging from $10^{2.3}$ to $10^{5.3} \mathrm{CFU} / \mathrm{g}$. Symbols:, $10^{2.3} ; \mathbf{D}, 10^{3.3} ; \mathbf{\Delta}$, $10^{4.1} ;>10^{5.3} \mathrm{CFU} / \mathrm{g}$. Asterisks show the counts enumerated with the most-probable-number method.

B. The initial dose dependency of the value for $N_{\max }$. The curve is expressed with equation 3 .

C. Prediction of the pathogen with a given initial dose $\left(10^{3.8}\right.$ $\mathrm{CFU} / \mathrm{g}$ ).

The temperatures studied were all $24^{\circ} \mathrm{C}$. Bars which show the standard deviations at data points are too small to appear in the figures. Growth curves in A and C are described and predicted with the extended logistic model. and $9.1 \log$ CFU/g with the initial doses of 2.3, 3.3, 4.1, and $5.3 \log \mathrm{CFU} / \mathrm{g}$, respectively.

The relationship between the initial dose $(I)$ and $N_{\max }$ was well fitted with a cubic equation with / (equation3).

$$
N_{\max }=0.030 I^{3}-0.52 I^{2}+3.2 I+2.2
$$

The equation could precisely describe the data points with the coefficient of regression of 0.999 (Fig. 1B). A growth curve of Salmonella in ground beef at a given value of / was then estimated with equation 3 to validate the polynomial model for $N_{\max }$. The value for $/$ in the experiment was measured to be $3.8 \mathrm{log} \mathrm{CFU} / \mathrm{g}$ and thus the $N_{\max }$ value was estimated to be $8.6 \mathrm{log} \mathrm{CFU} / \mathrm{g}$ with equation 3. Other parameter values in the growth model (equation 1) were the averages from the values of the curves studied in Fig. $1 \mathrm{~A}$, namely $r=0.64, m=0.58$, and $n=8.5$. With those parameter values, a growth curve was predicted and the curve was very close to the observed data (Fig. 1C): the RMSE value for the curve was as low as $0.12 \mathrm{log}$ CFU/g. This result showed that the polynomial model with I was applicable to estimate $N_{\max }$ at a constant temperature.

\section{Growth kinetics at constant temperatures}

The growth kinetics of Salmonella Enteritidis in ground beef was then studied at constant temperatures ranging from $8^{\circ} \mathrm{C}$ to $36^{\circ} \mathrm{C}$. Here the values for I were constant (3.2 log CFU/g) at these temperatures. Growth curves of Salmonella in ground beef at the constant temperatures were all sigmoidal and well described with the growth model (Fig. 2). No Salmonella growth was observed at $8^{\circ} \mathrm{C}$. Growth curves of NM in ground beef at those temperatures were also sigmoidal and well described with the growth model (data not shown).

The values of $r$ for Salmonella and NM in beef were well described with the square root model (Fig. 3). Linear regression lines for $r$ for Salmonella and NM were described by equations 4 and 5 , respectively. Here $T$ is temperature $\left({ }^{\circ} \mathrm{C}\right)$.

$$
\begin{aligned}
& \sqrt{r}=0.0401(T-3.47) \\
& \sqrt{r}=0.0259(T+5.56)
\end{aligned}
$$

The coefficients of determination for Salmonella and NM were 0.995 and 0.988 , respectively. The values for $N_{\max }$ for Salmonella and NM in beef were shown in Fig. 4. The values for $N_{\max }$ for Salmonella were described as a line broken at $28.3^{\circ} \mathrm{C}$, which is shown in equation 6 . 

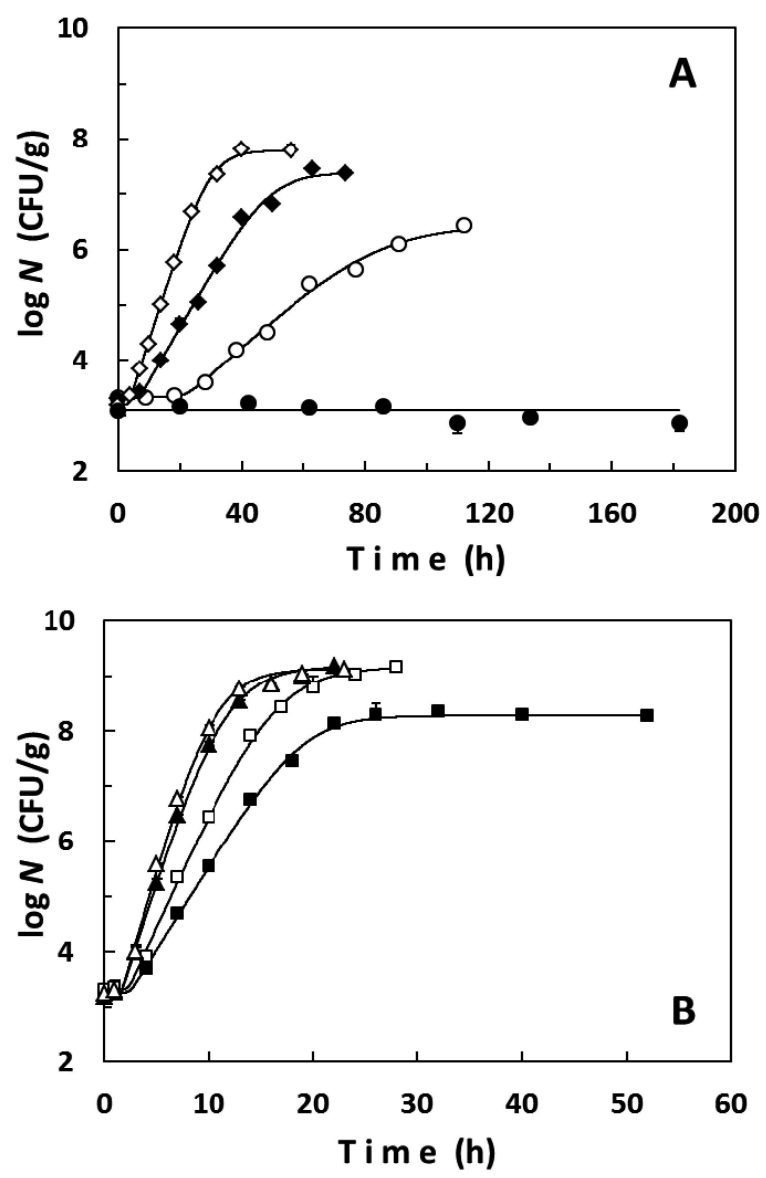

FIG. 2. Growth of Salmonella Enteritidis in raw ground beef at various temperatures ranging from $8^{\circ} \mathrm{C}$ to $36^{\circ} \mathrm{C}$.

Symbols: $\bigcirc 8^{\circ} \mathrm{C} ; \bigcirc, 12^{\circ} \mathrm{C} ;>, 16^{\circ} \mathrm{C} ; \diamond, 20^{\circ} \mathrm{C} ; \square, 24^{\circ} \mathrm{C} ; \square$, $28^{\circ} \mathrm{C} ; \boldsymbol{\Delta}, 32^{\circ} \mathrm{C} ; \Delta, 36^{\circ} \mathrm{C}$. The initial doses of the pathogen were constant $\left(10^{3.2} \mathrm{CFU} / \mathrm{g}\right)$. Bars which show the standard deviations at data points are too small to appear in the figures. Growth curves are described with the growth model.

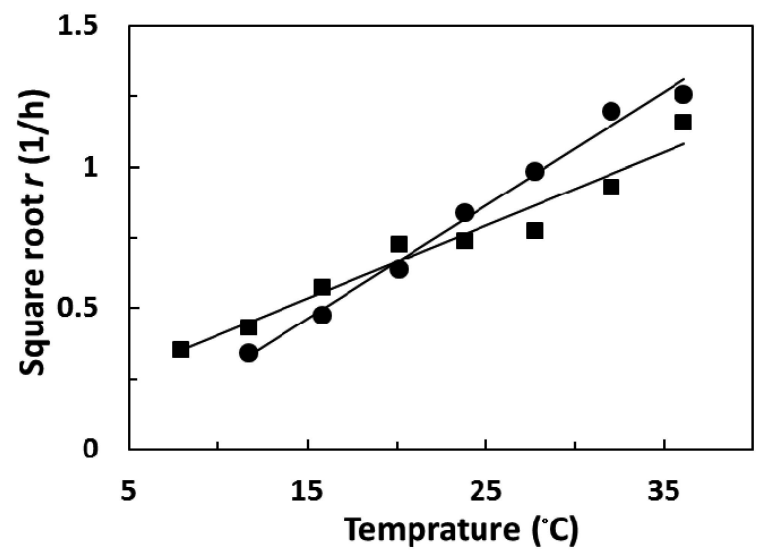

FIG. 3. Square root model for the rate constant of growth for the pathogen and NM. Symbols: O, Salmonella; $\mathbf{\square}$, NM. Straight lines are regression lines.

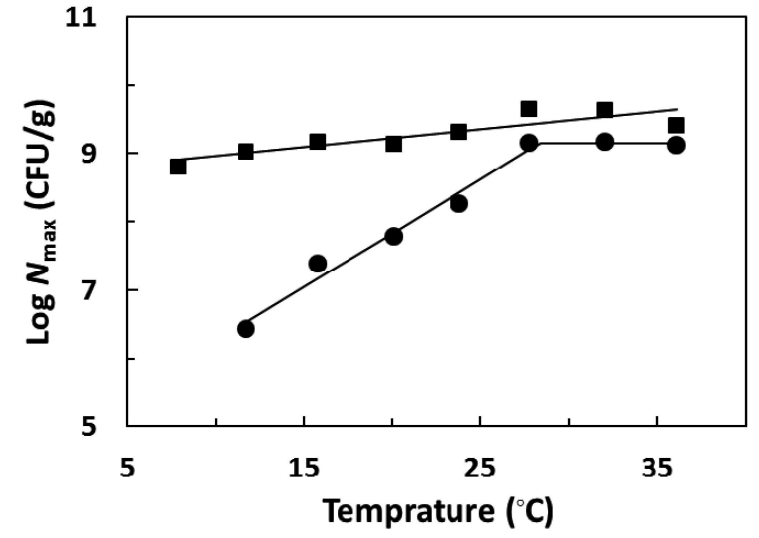

FIG. 4. The maximum population for Salmonella and NM at various constant temperatures. Symbols: Salmonella; NM. Lines are regression lines.

$$
\begin{aligned}
\log N_{\max } & =0.158 T+4.67 & & (12 \leq \mathrm{T}<28.3) \\
& =9.15 & & (28.3 \leq \mathrm{T} \leq 36)
\end{aligned}
$$

The values for $N_{\max }$ for $\mathrm{NM}$ were described as a straight line (equation 7).

$$
\log N_{\max }=0.0262 T+8.70
$$

The values for $m$ and $n$ for Salmonella at these temperatures were almost constant, being 0.47 and 6.4 as the averages, respectively. The values for $m$ and $n$ for NM were also almost constant, being 0.79 and 4.5, respectively.

\section{Prediction at dynamic temperatures}

Using the above equations (equations 4-7) and the averages of the parameters of the growth model (equation 1), growth curves of Salmonella and NM were predicted at dynamic temperatures. Here the value for I was constant (3.2 log CFU/g). The growth model could well predict both at dynamic temperatures (Fig. 5A, B). The RMSE values for Salmonella in Fig. 5A and $B$ were very low, which were 0.16 and 0.15 , respectively. The RMSE values for NM were also low, which were 0.27 and 0.34 , respectively. When the predicted populations were plotted to the measured ones, both were very close to each other for Salmonella and NM (Fig. 6). That is, most of the symbols in the figure were located on the line of equivalence or close to the line. Also, very high linearity between the predicted and measured populations was found for Salmonella and NM (Fig. 6); the values for the coefficient of determination for the pathogen and NM 

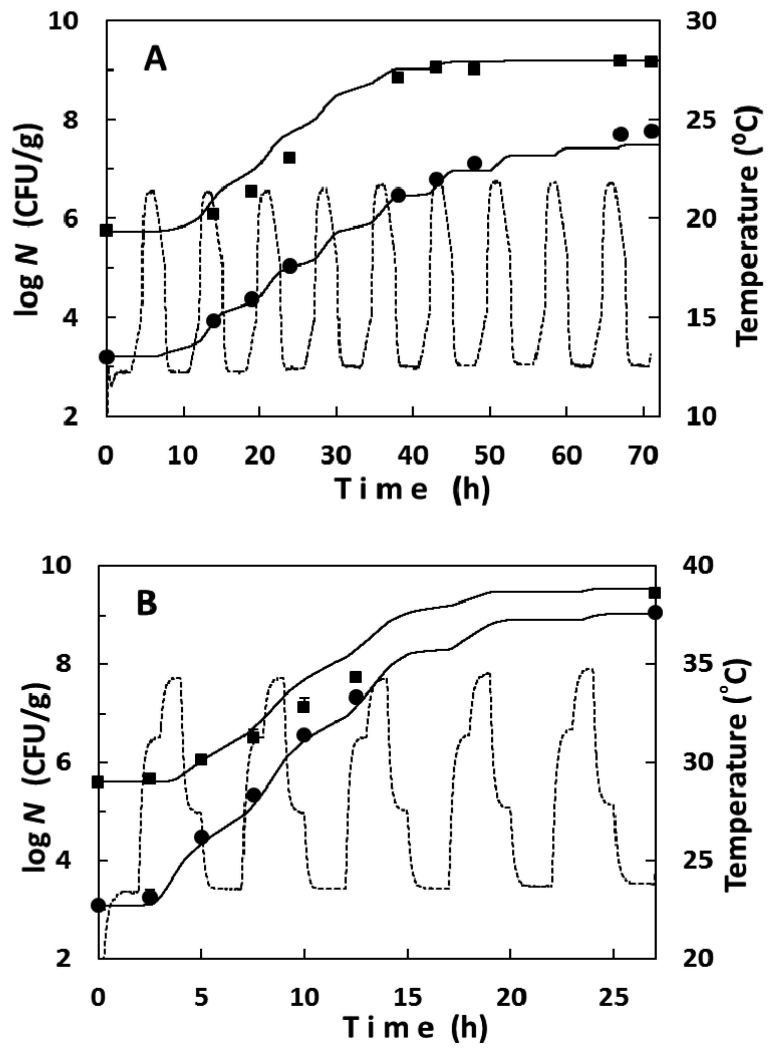

FIG. 5. Prediction of growth of Salmonella and NM in ground beef at dynamic patterns of temperature. Symbols: Salmonella; $\mathbf{\square}$, NM. Bars which show the standard deviations at data points are too small to appear in the figures. Curves are described with the growth model. A dotted line shows the measured temperature of ground beef.

were 0.999 and 0.987 , respectively.

\section{Competition between Salmonella and NM}

To investigate the relationship between Salmonella and $\mathrm{NM}$ in ground beef during the storage, the growth of NM in ground beef that was not inoculated with Salmonella, namely the original ground beef, was also studied at a given temperature $\left(36^{\circ} \mathrm{C}\right)$. When NM in the beef samples inoculated with the pathogen was compared to NM in the original beef sample without the pathogen, the growth of the former was a little suppressed (Fig. 7). The difference between the two types of NM was actually very small; the values for $N_{\max }$ were $9.4 \log \mathrm{CFU} / \mathrm{g}$ for the former and $9.8 \mathrm{log} \mathrm{CFU} / \mathrm{g}$ for the latter. However, a significant difference was found between them $(P<0.05)$, showing that the $N_{\max }$ value for NM in ground beef inoculated with Salmonella was lower than that without inoculation. Here the growth of Salmonella was also great with the $N_{\max }$ value of $9.1 \mathrm{log}$ CFU/g (Fig. 7). Similar observations were also found at a lower temperature such as $20^{\circ} \mathrm{C}$ (data

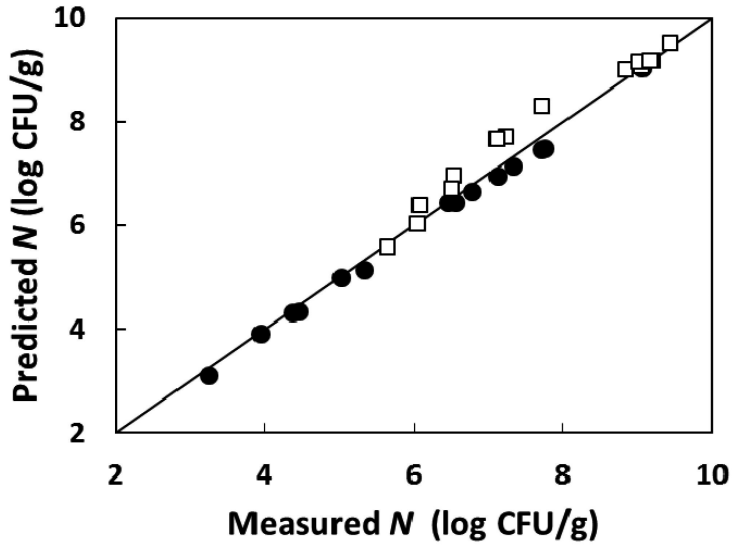

FIG. 6. Differences between the predicted and measured populations at the dynamic temperatures in Fig. 5. Symbols: -. Salmonella; $\square$, NM. The straight line is the line of equivalence.

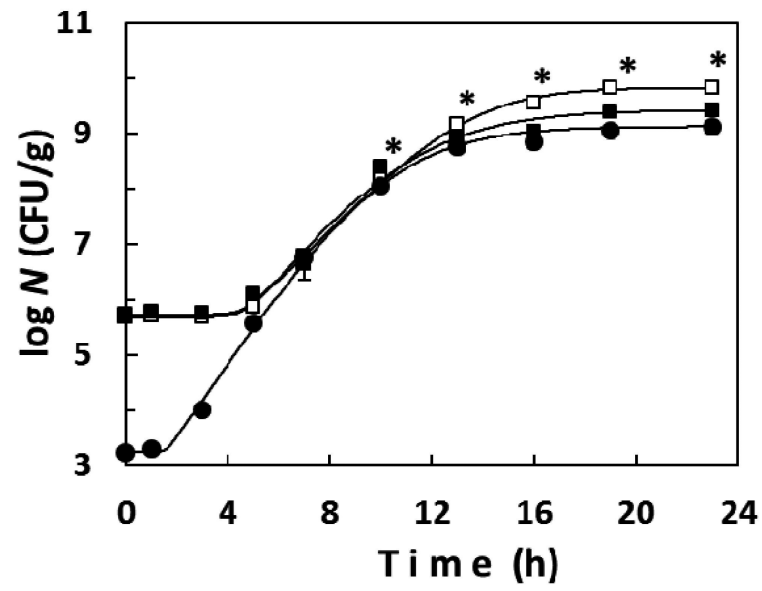

FIG. 7. Growth of NM in ground beef with and without Salmonella inoculation at $36^{\circ} \mathrm{C}$. Symbols; $\square$, NM in beef without Salmonella; $\mathbf{\square}$, NM in beef inoculated with Salmonella; Salmonella in inoculated beef. Bars which show the standard deviations at data points are too small to appear in the figure. Asterisks show the significant difference in the NM population between two types of ground beef by the $t$-test $(P<0.05)$. Curves are described with the growth model.

not shown). These results showed that NM and the pathogen in inoculated ground beef competed with each other during the growth.

\section{DISCUSSION}

The whole growth curves of Salmonella Enteritidis and NM in ground beef at various temperatures were kinetically studied in our study, different from previous studies (Mackey and Kerridge, 1988; Juneja et al., 
2009). The growth curves of Salmonella and NM in ground beef at dynamic temperatures in Fig. 5 were precisely predicted with the combination of the growth model, the square root model for $r$, and equations 6 and 7 for $N_{\max }$. This was also observed in the growth of the pathogen in ground chicken and the liquid egg products (Zaher and Fujikawa, 2011; Sakha and Fujikawa, 2012 and 2013). These results confirmed that the system of the models and the equations using a given initial dose were usable for the prediction of the growth of Salmonella and NM at various patterns of temperature.

Many researchers have studied the growth of a foodborne pathogen competing with NM in food using mathematical models. Gimenez and Dalgaard (2004) also developed a competition model for Listeria monocytogenes and lactic acid bacteria, which was a log-transformed logistic model. In this model, the equation for $L$. monocytogenes includes a term of suppression with lactic acid bacteria and vice versa. Le Marc et al. (2009) developed a competition model by introducing the concept of critical population density for lactic acid bacteria against a competing bacterium ( $S$. aureus). Breidit and Fleming (1998) also modeled the competitive growth of $L$. monocytogenes and Lactococcus lactis by modeling the concentration of lactic acid produced and the $\mathrm{pH}$ of vegetable broth. These models were primary models modified for microbial competition and seemed to be specific to the species of concern.

On the other hand, the Lotka-Volterra (LV) model is a very well-known and general model for the expression of competition between two species in ecology (Vandermeer and Goldber, 2003). Dens et al. (1999) proposed a microbial competition model constructed with the Baranyi model and the LV model and showed the characteristics of the competition model by mathematical simulations. For the LV model, however, one needs to know the growth data of each microbe in monoculture in a given sterile food in advance (Fujikawa et al., 2014). With such monoculture data, a growth model coupled with the LV model can describe and predict the growth curves of the competitors in mixed culture (Fujikawa et al., 2014). This means that the LV model is limited in application to the growth of a microbe in actual raw food contaminated with NM, because it is generally very hard to sterilize the raw food without any changes in the food. Therefore we now think that the present system consisting of the growth model, the square root model, and the equations for $N_{\max }$ would be a practical system applicable to actual food.

The freezing of the beef samples at $-40^{\circ} \mathrm{C}$ did not affect the survival of NM in the samples in this study.
That is, the initial populations of NM of the samples throughout the present study were almost constant, ranging from 5.6 to $5.9(\log \mathrm{CFU} / \mathrm{g})$ with the average of $5.7 \pm 0.07(\log C F U / g)$. Also, there was no decrease in the NM population of the samples as the freezing period proceeded during the study (data not shown).

The regression line of the rate constant for Salmonella in the square root plot intersected with the regression line for NM in Fig. 3. The temperature of intersection was calculated to be $20.0^{\circ} \mathrm{C}$. This meant that the growth of the pathogen at the temperatures above $20.0^{\circ} \mathrm{C}$ would overcome the growth of NM in the beef sample and vice versa. The same phenomenon between Salmonella and NM was observed in our previous study with ground chicken (Zaher and Fujikawa, 2011). Competition between the pathogen and NM, which was quantitatively observed in Fig. 7, might be also related to the above relationship seen in Fig. 3. On the other hand, the $N_{\max }$ values for Salmonella at various constant temperatures were suppressed at temperatures $>28.3^{\circ} \mathrm{C}$ (Fig. 4). This phenomenon might be also derived from the competition between the pathogen and NM.

The growth characteristics of Salmonella Enteritidis in ground beef at various initial doses shown in Fig. 1A were the same as in ground chicken and unpasteurized liquid egg in our previous studies (Zaher and Fujikawa, 2011; Sakha and Fujikawa, 2012 and 2013). That is, the growth rates of the pathogen were similar to each other, while the $N_{\max }$ values for the pathogen were dependent on the initial doses. On the other hand, the growth of the pathogen in sterile food were quite different; the $N_{\max }$ values with various initial doses of the pathogen were much higher and constant (Zaher and Fujikawa, 2011; Sakha and Fujikawa, 2012).

The present study confirmed that the growth model can successfully describe and predict the growth of Salmonella inoculated at a given initial population in raw ground beef at various temperatures. The growth data obtained here would be valuable information for the regulation of the pathogen in ground beef. It was also confirmed that the $N_{\max }$ value for Salmonella in ground beef was successfully expressed with the polynomial equation 3 with I (Fig.1C). These findings would lead to a hypothesis that the value for $N_{\max }$ might be expressed by a polynomial equation with the initial dose and temperature. If so, a model system consisting of our growth model, the polynomial equation for $N_{\max }$, and the square root model for $r$ might describe and predict the growth of the pathogen in ground beef at given values for I and temperature. In the near future, we would like to study if this system can predict such growth of the pathogen. 


\section{ACKNOWLEDGEMENTS}

The authors thank Mr. M. Z. Sakha and Mr. H. Rokugawa for their technical help and advice.

\section{REFERENCES}

Anonymous (2004) Standard method of analysis in food safety regulation. Japan Food Hygiene Association, Tokyo, Japan.

Arthur, T.M., Brichta-Harhay, D.M., Bosilevac, J.M., Guerini, M.N., Kalchayanand, N., Wells, J.E., Shackelford, S.D., Wheeler, T.L., and Koohmaraie, M. (2008) Prevalence and characterization of Salmonella in bovine lymph nodes potentially destined for use in ground beef. J. Food Prot., 71, 1685-1688.

Ayres, J.C. (1955) Microbiological implications in the handling, slaughtering, and dressing of meat animals. Adv. Food. Res., 6,109-161.

Breidt, F., and Fleming, H.P. (1998) Modeling of the competitive growth of Listeria monocytogenes and Lactococcus lactis in vegetable broth. Appl. Environ. Microbiol., 64, 3159-3165.

CDC (Centers for Disease Control and Prevention) (1995) Outbreak of Salmonella Serotype Typhimurium Infection Associated with Eating Raw Ground Beef - Wisconsin, 1994. MMWR., 44, 905-909. http://www.cdc.gov/mmwr/ preview/mmwrhtml/00039761.htm.

CDC (Centers for Disease Control and Prevention) (2006) Multistate outbreak of Salmonella Typhimurium infections associated with eating ground beef-United States, 2004. MMWR., 55, 180-182. http://www.cdc.gov/mmwr/ preview $/ \mathrm{mmw}$ rhtml/mm5507a4.htm.

CDC (Centers for Disease Control and Prevention) (2012) Multistate outbreak of Salmonella Enteritidis infections linked to ground beef (Final Update). http://www.cdc.gov/ salmonella/enteritidis-07-12/index.html.

CDC (Centers for Disease Control and Prevention) (2013) Multistate outbreak of human Salmonella Typhimurium infections linked to ground beef (final update). http://www. colc.gov/salmonella/typhimurium-01-13/.

D'Aoust, J.Y. (1991) Psychrotrophy and foodborne Salmonella. Int. J. Food Microbiol., 13, 207-215.

Dens, E.J., Vereechen, K.M., and Van Impe, J.F. (1999) A prototype models structure for mixed microbial populations in homogeneous food products. J. Theor. Biol., 201, 159-170.

Fujikawa, H., Kai, A., and Morozumi, S. (2003) A new logistic model for bacterial growth. J. Food Hyg.Soc. Japan, 44, 155-160.

Fujikawa, H., Kai, A., and Morozumi, S. (2004) A new logistic model for Escherichia coli at constant and dynamic temperatures. Food Microbiol., 21, 501-509.

Fujikawa, H., and Kano, Y. (2009) Development of a program to fit data to a new logistic model for microbial growth. Biocont. Sci., 14, 83-86.

Fujikawa, H., Munakata, K., and Sakha, M.Z. (2014) Development of a competition model for microbial growth in mixed culture. Biocont. Sci., 19, 61-71.

Gimenez, B., and Dalgaard, P. (2004) Modelling and predicting the simultaneous growth of Listeria monocytogenes and spoilage microorganisms in cold-smoked salmon. J. Appl. Microbiol., 96, 96-109.

Hendriksen, R.S., Vieira, A.R., Karlsmose, S., Danilo, M.A., Wong, L.F., Jensen, A.B., Wegener, H.C., and Aarestrup, F.M. (2011) Global Monitoring of Salmonella serovar distri- bution from the World Health Organization Global Foodborne Infections Network Country Data Bank: Results of Quality Assured Laboratories from 2001 to 2007. Foodborne Pathog. Dis., 8, 887-900.

IDSC (Infectious Disease Surveillance center) (2014) Fifteen most common Salmonella serovars from human sources. (Prefectural/municipal public health institutes and health centers) Data Based on the reports received before February 4, 2014. http://idsc.nih.go.jp/iasr/virus/graph/ salm0509.pdf.

Jackson, B.R., Griffin, P.M., Cole, D., Walsh, K., and Chai, S.J. (2013) Outbreak-associated Salmonella enterica serotypes and food commodities, United States, 19982008. Emerg. Infect. Dis., [serial online] 19. http://dx.doi. org/10.3201/eid1908.121511.

Juneja, V.K., Melendres, M.V., Huang, L., Subbiah, J., and Thippareddi, H. (2009) Mathematical modeling of growth of Salmonella in raw ground beef under isothermal conditions from 10 to $45^{\circ} \mathrm{C}$. Int. J. Food Microbiol., 131, 106-111.

Le Marc, Y., Valik, L., and Medved' ová, A. (2009) Modeling the effect of the starter culture on the growth of Staphylococcus aureus in milk. Int. J. Food Microbiol., 129, 306-311.

Mackey, B.M., and Kerridge, A.L. (1988) The effect of incubation temperature and inoculum size on growth of salmonellae in minced beef. Int. J. Food Microbiol., 6, 57-65.

Majowicz, S.E., Musto, J., Scallan, E., Angulo, F. J., Kirk, M., O' Brien, S. J., Jones, T. F., Fazil, A., and Hoekstra, R.M. (2010) The global burden of nontyphoidal Salmonella gastroenteritis. Clin. Infect. Dis., 50, 882-889.

McMeekin, T.A., Olley, J.N., Ross, T., and Ratkowsky, D.A. (1993) Predictive Microbiology: Theory and Application. Taunton (UK): Research Studies Press.

Moller, C.O.A., Ilg, Y., Aabo, S., Christensen, B.B., Dalgaard, P., and Hansen, T.B. (2013) Effect of natural microbiota on growth of Salmonella spp. in fresh pork-a predictive microbiology approach. Food Microbiol., 34, 284-295.

Ratkowsky, D.A., Olley, J., McMeekin, T.A., and Ball, A. (1982) Relationship between temperature and growth rate of bacterial cultures. J. Bacteriol., 149, 1-5.

Roels, T. H., Frazak, P., Kazmierczak, J. J., Mackenzie, W. R., Proctor, M. E., Kurzynski, T., and Davis, J. P. (1997) Incomplete sanitation of a meat grinder and ingestion of raw ground beef: contributing factors to a large outbreak of Salmonella Typhimurium infection. Epidemiol. Infect., 119, 127-34.

Sakha, M. Z., and Fujikawa, H. (2012) Growth characteristics of Salmonella Enteritidis in pasteurized and unpasteurized liquid egg products. Biocontrol Sci., 17, 183-190.

Sakha, M. Z., and Fujikawa, H. (2013) Prediction of Salmonella Enteritidis growth in pasteurized and unpasteurized liquid egg products with a growth Model. Biocontrol Sci., 18, 89-93.

USDA (US Department of Agriculture) and FSIS (Food Safety and Inspection Service). (2012) Progress report on Salmonella and Campylobacter testing of raw meat and poultry products, CY 1998-2012. http://www.fsis.usda. gov/wps/portal/fsis/ topics/data-collection-and-reports/ microbiology/annual-progress-reports/2012-annual-report.

Vandermeer, J.H and Goldber, G.D.E. (2003) Population Ecology: First Principles. Princeton University Press, Princeton, New Jersey.

Zaher, S.M., and Fujikawa, H. (2011) Effect of native microflora on the growth kinetics of Salmonella Enteritidis strain 04-137 in raw ground chicken. J. Food Prot., 74, 735-742. 